\title{
Cluster Algebras for Feynman Integrals
}

\author{
Dmitry Chicherin $\odot^{*}$ and Johannes M. Henn $\odot^{\dagger}$ \\ Max-Planck-Institut für Physik, Werner-Heisenberg-Institut, 80805 München, Germany \\ Georgios Papathanasiou $\odot^{\ddagger}$ \\ DESY Theory Group, DESY Hamburg, Notkestrasse 85, 22607 Hamburg, Germany
}

(Received 22 December 2020; accepted 28 January 2021; published 5 March 2021)

\begin{abstract}
We initiate the study of cluster algebras in Feynman integrals in dimensional regularization. We provide evidence that four-point Feynman integrals with one off-shell leg are described by a $C_{2}$ cluster algebra, and we find cluster adjacency relations that restrict the allowed function space. By embedding $C_{2}$ inside the $A_{3}$ cluster algebra, we identify these adjacencies with the extended Steinmann relations for six-particle massless scattering. The cluster algebra connection we find restricts the functions space for vector boson or Higgs plus jet amplitudes and for form factors recently considered in $\mathcal{N}=4$ super Yang-Mills. We explain general procedures for studying relationships between alphabets of generalized polylogarithmic functions and cluster algebras and use them to provide various identifications of one-loop alphabets with cluster algebras. In particular, we show how one can obtain one-loop alphabets for five-particle scattering from a recently discussed dual conformal eight-particle alphabet related to the $G(4,8)$ cluster algebra.
\end{abstract}

DOI: 10.1103/PhysRevLett.126.091603

Introduction.-Recent years have seen the emergence of unexpected mathematical structures in scattering amplitudes, especially so in $\mathcal{N}=4$ supersymmetric Yang-Mills (SYM). Planar loop integrands in this theory are, in principle, known to all loop orders [1], with the on-shell data entering the former being described by a positive Grassmannian [2]. Furthermore, the integrand has a dual geometric description [3]. There is considerable evidence that cluster algebras play an important role for the amplitudes, both at the level of the loop integrand [4] as well as for the functions obtained after integration [5]. For example, planar six- and seven-gluon scattering amplitudes appear to be governed by the finite $A_{3}$ and $E_{6}$ cluster algebras, respectively. This suggests that their function space is a certain set of generalized polylogarithms, which is the starting point for the bootstrap program [6,7]. Further constraints come from the absence of discontinuities in overlapping channels, the (extended) Steinmann relations [8-10], which are closely related to cluster adjacency properties [11,12]. These findings have been instrumental for bootstrapping amplitudes to very high loop orders; see, e.g., [13-16], and the review [17].

Published by the American Physical Society under the terms of the Creative Commons Attribution 4.0 International license. Further distribution of this work must maintain attribution to the author(s) and the published article's title, journal citation, and DOI. Funded by SCOAP ${ }^{3}$.
How general is the appearance of cluster algebras in quantum field theory? On the one hand, all known cases are related to planarity and concern finite parts of amplitudes in $\mathcal{N}=4$ SYM, which have additional symmetries [18-20]. On the other hand, it motivates us that several structures initially found in $\mathcal{N}=4 \mathrm{SYM}$, such as insights into the transcendental structure of Feynman integrals [21], their evaluation [22], and dealing with their analytic properties and identities between them [23], are by now common tools in generic quantum field theories, as reviewed in Refs. $[24,25]$. In this Letter, we initiate a study of cluster algebras in Feynman integrals in $D=4-2 \epsilon$ dimensions, without relying on extra symmetries.

Cluster algebras and associated function spaces.Cluster algebras [26-29] (see [30-33] for introductory articles) are commutative algebras equipped with a distinguished set of generators $a_{i}$, the cluster $\mathcal{A}$ coordinates, grouped into overlapping subsets $\boldsymbol{a} \equiv\left\{a_{1}, \ldots, a_{d}\right\}$ of rank $d$, the clusters. Starting from an initial cluster, they may be constructed recursively by a mutation operation on the cluster coordinates. They may be generalized to contain frozen coordinates $\left\{a_{d+1}, \ldots, a_{d+m}\right\}$ that do not change under mutations.

How the cluster coordinates transform under a mutation is encoded in an integer $(d+m) \times d$ exchange matrix $B$, whose components we will denote as $b_{i j}$. Restricting to the components with $i, j \leq d$ corresponds to the principal part of $B$, which must be skew symmetrizable. Then, mutating a cluster $(\boldsymbol{a}, B)$ along the $k$ th variable, with $1 \leq k \leq d$, we obtain the new cluster $\left(\boldsymbol{a}^{\prime}, B^{\prime}\right)$, whose exchange matrix $B^{\prime}$ is related to the previous one by 
$b_{i j}^{\prime}= \begin{cases}-b_{i j}, & \text { if } i=k \text { or } j=k, \\ b_{i j}+\left[-b_{i k}\right]_{+} b_{k j}+b_{i k}\left[b_{k j}\right]_{+} & \text {otherwise }\end{cases}$

where $[x]_{+}=\max (0, x)$. The cluster coordinates $a_{i}$ are unchanged for $i \neq k$, and $a_{k}$ is mutated according to

$$
a_{k}^{\prime}=a_{k}^{-1}\left(\prod_{i=1}^{d+m} a_{i}^{\left[b_{i k}\right]_{+}}+\prod_{i=1}^{d+m} a_{i}^{\left[-b_{i k}\right]_{+}}\right)
$$

The $C_{2}$ cluster algebra will play a prominent role in this Letter. For the coefficient-free case $m=0$, it is defined by

$$
B=\left(\begin{array}{cc}
0 & 1 \\
-2 & 0
\end{array}\right)
$$

Then, it is easy to show that under a mutation (1) $B$ changes only by a sign, and, thus, the corresponding cluster transformation (2) simplifies to

$$
a_{m+1} a_{m-1}= \begin{cases}1+a_{m} & \text { if } m \text { is odd } \\ 1+a_{m}^{2} & \text { if } m \text { is even }\end{cases}
$$

where $\left\{a_{1}, a_{2}\right\}$ are the $\mathcal{A}$ coordinates of the initial cluster, $a_{3}=a_{1}^{\prime}$, and so on. The coordinates obtained in this manner are shown in the exchange graph in Fig. 1, where clusters $\left\{a_{i}, a_{i+1}\right\}$ are represented by vertices and the mutations relating them by edges. The circle topology indicates that mutating six times takes us back to where we started, $a_{i+6}=a_{i}$.

Below, we will also find it useful to consider an alternative set of coordinates, called cluster $\mathcal{X}$ coordinates [34], which are defined as

$$
x_{i} \equiv \prod_{l=1}^{d+m} a_{l}^{b_{l i}}, \quad i=1, \ldots, d
$$

The $\mathcal{A}$-coordinate mutation (2) and Eq. (5) imply a relation between the associated $\mathcal{X}$ coordinates, which may be written directly as

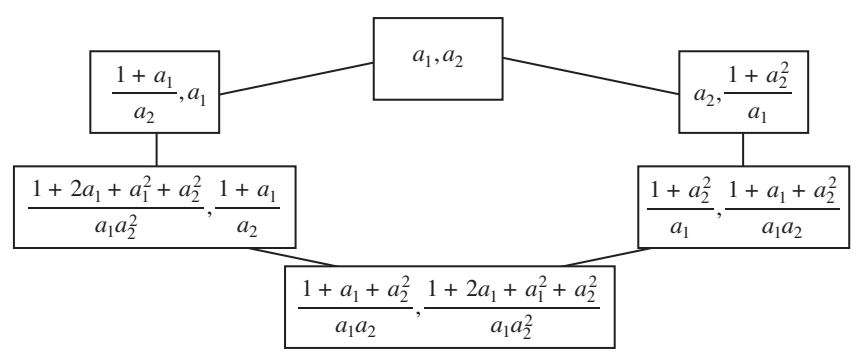

FIG. 1. The exchange graph of the $C_{2}$ cluster algebra, with cluster coordinates ordered as $a_{i}, a_{i+1}$.

$$
x_{i}^{\prime}= \begin{cases}1 / x_{i} & k=i, \\ x_{i}\left(1+x_{k}^{-\operatorname{sgn}\left(b_{k i}\right)}\right)^{-b_{k i}} & k \neq i .\end{cases}
$$

In fact, the latter provide another way of defining cluster algebras (more precisely, cluster Poisson varieties): One may start with the $\mathcal{X}$ coordinates and the principal part of $B$ of the initial cluster and obtain all other clusters by virtue of the $\mathcal{X}$ coordinate mutation (6).

Let us now associate a natural function space to a cluster algebra, given a set $\left\{a_{i}\right\}$ of $\mathcal{A}$ (or, similarly, $\mathcal{X}$ ) coordinates. A cluster (polylogarithm) function $f$ [35] of (transcendental) weight $w$ has the defining property that its differential has the form

$$
d f^{(w)}=\sum_{i} f_{i}^{(w-1)} d \log a_{i}
$$

where the $f_{i}$ are again cluster functions, of weight $(w-1)$. The iterative definition starts with the weight zero function, which is a constant. From this, it follows that cluster functions of weight $w$ can be expressed as $w$-fold Chen iterated integrals [36].

The definition (7) is very similar to canonical differential equations satisfied by certain classes of Feynman integrals [22]:

$$
d \mathbf{f}(\vec{z} ; \epsilon)=\epsilon\left[\sum_{i} \mathbf{A}_{i} d \log \alpha_{i}(\vec{z})\right] \mathbf{f}(\vec{z} ; \epsilon),
$$

where $\mathbf{f}$ is a basis of Feynman integrals under consideration, $\mathbf{A}_{i}$ are constant matrices, the $\alpha_{i}$ are algebraic functions of the kinematic variables $\vec{z}$, and $d=\sum_{j} d z_{j} \partial_{z_{j}}$. Solving Eq. (8) as a series in $\epsilon$ yields Chen iterated integrals, with the weight corresponding to the order in $\epsilon$. Moreover, the symbol [23] of the answer, which amounts to the solution modulo integration constants, can be read off from Eq. (8). The set of $\alpha$ 's is called the alphabet, and its elements are called letters.

In the following, we wish to study whether the alphabets of certain Feynman integrals coincide with the $\mathcal{A}$ or $\mathcal{X}$ coordinates of some cluster algebra.

$\mathrm{C}_{2}$ cluster algebra and four-particle scattering with one off-shell leg.-We consider four-particle scattering processes with one off-shell leg and massless internal lines; cf. Fig. 2. This kinematic situation has many important physical applications. It applies to helicity amplitudes of a Higgs boson and three partons $[37,38]$ or of a vector boson
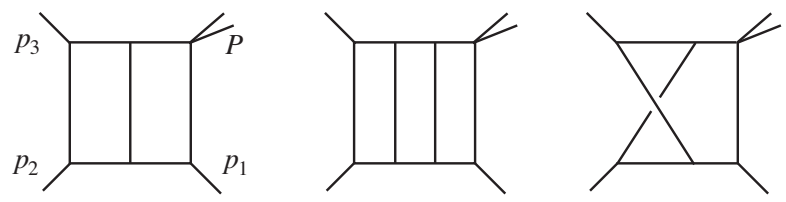

FIG. 2. Examples of known two- and three-loop four-point integrals with one off-shell leg, $P^{2} \neq 0$. 
and three partons [39]. Interestingly, it has been observed that the Higgs amplitudes bear a close resemblance to $\mathcal{N}=4$ SYM form factors of a composite operator inserted into three on-shell states [40]. What is more, very recently, an integrability description for form factors in $\mathcal{N}=4 \mathrm{SYM}$ was derived [41], which provides valuable all-loop and even nonperturbative information.

Denoting the Lorentz invariants by $z_{1}=s / P^{2}$ and $z_{2}=t / P^{2}$, with $s=2 p_{1} \cdot p_{2}$ and $t=2 p_{2} \cdot p_{3}$, we note that all known Feynman integrals (i.e., all planar and nonplanar two-loop integrals $[42,43]$ and certain planar three-loop integrals [44]; see Fig. 2) in this kinematics can be expressed in terms of the following alphabet (to all orders in $\epsilon$ ):

$$
\Phi_{2 \mathrm{DHPL}}=\left\{z_{1}, z_{2}, z_{3}, 1-z_{1}, 1-z_{2}, 1-z_{3}\right\},
$$

with $z_{1}+z_{2}+z_{3}=1$. The associated class of functions, dubbed two-dimensional harmonic polylogarithms (2DHPL), is well studied in the physics literature $[38,45,46]$.

We now show that, surprisingly, the 2DHPL alphabet (9) is equivalent to the $C_{2}$ alphabet given in Fig. 1. To see this, one applies the mapping

$$
z_{1}=-\frac{a_{2}^{2}}{1+a_{1}}, \quad z_{2}=-\frac{1+a_{1}+a_{2}^{2}}{a_{1}\left(1+a_{1}\right)} .
$$

We make a number of interesting observations.

1. The cluster $\mathcal{X}$ coordinates are useful for choosing a particular polylogarithmic representation of the functions and for writing down functional identities $[35,38,45,47]$. For example, if $x$ is an $\mathcal{X}$ coordinate, then $\mathrm{Li}_{n}(-x)$ is a cluster function, as can be understood from Eq. (6). In Ref. [35], an algorithm for constructing a basis of $A_{n}$ cluster functions at any weight is given. Moreover, we note that the $C_{2}$ cluster mutations generate automorphisms of the 2DHPL alphabet.

2. In certain functions in $\mathcal{N}=4 \mathrm{SYM}$, the interesting property of cluster adjacency [11] has been observed, which in the language of differential equations (8) translates to the statement that $\mathbf{A}_{i} \cdot \mathbf{A}_{j}=0$ if $\alpha_{i}$ and $\alpha_{j}$ do not appear together in any cluster. Note that this proves the adjacency property to all orders in $\epsilon$. Inspecting the differential equations for the two- and three-loop planar integrals of Refs. [42,44], we observe that they obey a subset of $C_{2}$ adjacency conditions, namely,

$$
\mathbf{A}_{i} \cdot \mathbf{A}_{j}=0 \quad \text { for } i, j \in\{1,3,5\} \quad \text { with } \quad i \neq j .
$$

Equation (11) also holds for the non-planar integrals of [43], as was first noticed in [48]. In terms of the equivalent alphabet (9), this implies that the letters $1-z_{i}$ and $1-z_{j}$ for $i \neq j$ never appear next to each other in a symbol. The adjacency conditions we observe significantly reduce the
TABLE I. Dimension of the $C_{2}$ cluster functions space (modulo transcendental constants), after constraints.

\begin{tabular}{lcccccccc}
\hline \hline Weight & 1 & 2 & 3 & 4 & 5 & 6 & 7 & 8 \\
\hline First entry condition & 3 & 12 & 45 & 165 & 597 & 2143 & 7653 & 27241 \\
Adjacency constraint & 3 & 12 & 42 & 138 & 438 & 1362 & 4182 & 12738 \\
\hline \hline
\end{tabular}

dimension of the space containing the aforementioned integrals, as shown in Table I.

Furthermore, the adjacency property extends to rational functions [12], as can be seen by inspecting, for example, the two-loop amplitudes of Refs. [40,49]. This is obvious, because the latter contain poles only at $z_{i}=0$.

3 . We can interpret the observed adjacency in the following way: As shown in Fig. $3, C_{2}$ is the parity-invariant surface of the $A_{3}$ cluster algebra, relevant for six-gluon amplitudes in $\mathcal{N}=4$ SYM [50]. On the latter surface, corresponding to $\Delta \equiv(u+v+w-1)^{2}-4 u v w=0$, where $u$, v, and $w$ are the dual conformal cross-ratios parametrizing the kinematics, only parity-even combinations of the $A_{3} \mathcal{A}$ coordinates are relevant. Choosing them as

$$
\begin{aligned}
& a_{1}=\sqrt{\frac{u}{v w}}, \quad a_{3}=\sqrt{\frac{w}{u v}}, \quad a_{5}=\sqrt{\frac{v}{u w}}, \\
& a_{2}=\sqrt{\frac{1-v}{v}}, \quad a_{4}=\sqrt{\frac{1-u}{u}}, \quad a_{6}=\sqrt{\frac{1-w}{w}},
\end{aligned}
$$

where $\left\{a_{1}, a_{2}\right\}$ are associated to the leftmost equatorial cluster in Fig. 3, $\left\{a_{6}, a_{1}\right\}$ to the left front cluster, and so on, we find that they satisfy exactly the $C_{2}$ mutation rule, Eq. (4), on the $\Delta=0$ surface. By virtue of Eq. (12), we recognize that the adjacency restrictions (11) precisely correspond to the extended Steinmann relations $[9,10]$ (i.e., applying to any consecutive entries in the symbol) for six-particle massless scattering $[52,53]$.

4. Finally, let us comment on the massless limit $P^{2} \rightarrow 0$. Denoting $z=t / s$, Eq. (9) reduces to the alphabet $\{z, 1+z\}$ in the limit. This is consistent with the fact that all currently known Feynman integrals in this kinematics (up to three

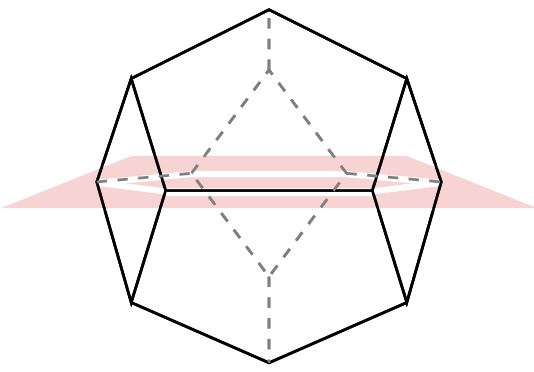

FIG. 3. The intersection of the exchange graph of the $A_{3}$ cluster algebra with the parity-invariant plane (in pink) is shown in white. It can be identified with the $C_{2}$ cluster exchange graph shown in Fig. 1. 
loops, planar and nonplanar) satisfy differential equations (8) with this alphabet [54-56]. It is interesting to note that one can obtain this on-shell four-particle alphabet from an $A_{1}$ cluster algebra with one frozen variable.

Matching function spaces and cluster algebras, and applications.-There are many other cases where Feynman integrals evaluate to generalized polylogarithmic functions that are characterized by an alphabet. Are any of them associated to cluster algebras? If so, how can we find this relation?

We use the following procedure to find embeddings of alphabets into cluster algebras. Let $\vec{\alpha}$ be an alphabet depending on $d$ variables $z_{1}, \ldots, z_{d}$ in a rational way. We would like to express all letters $\vec{\alpha}$ in terms of the letters $\vec{\beta}$ of a candidate cluster alphabet (with more or equal number of letters):

$$
d \log \left(\alpha_{i}\right)=\sum_{j} n_{i j} d \log \left(\beta_{j}\right)
$$

where $n_{i j}$ are integers. We order the letters $\vec{\alpha}$ according to their "complexity," namely, the number of $z$ variables they depend on. Parsing though the transformations with small integer coefficients $n_{i j}$, we identify those which are consistent with the one-variable letters. Then we further restrict the set of allowed transformations demanding that twovariable letters also factorize into the cluster letters, and so on for letters of higher complexity. This yields a host of novel matchings, which are indicated in Table II with the $\subset$ sign.

Let us illustrate this for the two-mass-easy box integral family with massive legs $p_{1}$ and $p_{3}$. The corresponding tenletter alphabet is given by [58]

$$
\begin{gathered}
\Phi_{2 m e}=\left\{s, t, p_{1}^{2}, p_{3}^{2}, s-p_{1}^{2}, s-p_{3}^{2}, t-p_{1}^{2}, t-p_{3}^{2},\right. \\
\left.s t-p_{1}^{2} p_{3}^{2}, s+t-p_{1}^{2}-p_{3}^{2}\right\} .
\end{gathered}
$$

The change of variables

$$
z_{1}=-\frac{p_{1}^{2}}{s}, \quad z_{2}=-\frac{t}{p_{3}^{2}}, \quad z_{3}=\frac{t-p_{1}^{2}}{s-p_{3}^{2}}
$$

brings it to a simpler form:

$$
\begin{aligned}
\Phi_{2 m e} \approx & \left\{z_{1}, z_{2}, z_{3}, 1+z_{1}, 1+z_{2}, 1+z_{3},\right. \\
& \left.z_{1}-z_{2}, z_{1}-z_{3}, z_{2}-z_{3}\right\},
\end{aligned}
$$

TABLE II. One-loop integrals related to cluster algebras.

\begin{tabular}{lccc}
\hline \hline Integral family & $\begin{array}{c}\text { Number of } \\
\text { variables }\end{array}$ & $\begin{array}{c}\text { Number of } \\
\text { letters }\end{array}$ & $\begin{array}{c}\text { Cluster } \\
\text { algebra }\end{array}$ \\
\hline $\begin{array}{c}\text { One-loop Bhabha } \\
\text { scattering [57] }\end{array}$ & 2 & 8 & $\subset A_{3}, \lim C_{3}$ \\
Two-mass-easy box & 3 & $7(9)$ & $\subset A_{3}$ \\
Two-mass-hard box & 3 & 10 & $\subset C_{3}, \lim D_{4}$ \\
Three-mass box & 4 & 16 & $\subset C_{4}$ \\
\hline \hline
\end{tabular}

where we dropped a trivial mass scale. This nine-letter alphabet is equivalent to the cluster $A_{3}$ alphabet.

We can find further identifications by considering degenerations of cluster algebra alphabets with fewer variables. What we mean by this is to consider the subalphabet obtained at a hypersurface $\alpha_{i}=0$ (for some $i$ ). We have already seen an example of this, when going from $C_{2}$ to $A_{1}$. We note that another degeneration of $C_{2}$ yields the important alphabet $\{z, 1+z, 1-z\}$ corresponding to harmonic polylogarithms [59]. The results of our heuristic search with this method are denoted in Table II by "lim."

The identifications of alphabets discussed so far are valid to all orders in the dimensional regularization parameter $\epsilon$. However, when computing finite physical quantities, often simplifications occur, and a reduced alphabet is sufficient to describe the answer. Moreover, it could be that cluster algebras appear, in general, only for integer dimensions. For these reasons, we find it interesting to look for cluster algebras for finite Feynman integrals or when truncating the $\epsilon$ expansion. It is known that the (dual conformal) sixdimensional hexagon integral corresponds to the $A_{3}$ cluster algebra. Here find that its one-mass version $[60,61]$, which has 20 letters and four dimensionless variables, corresponds to the $D_{4}$ cluster algebra. Likewise, we find that a "two-mass-easy" hexagon [61] can be embedded into the $D_{5}$ cluster algebra. See Table III.

So far, we have discussed finite cluster algebras; however, in $\mathcal{N}=4 \mathrm{SYM}$, also infinite cluster algebras are seen to play a role. For example, the infinite Grassmannian $G(4,8)$ algebra is expected to govern dual conformal eightparticle scattering, generalizing the six- and seven-particle case, with $A_{3} \sim G(4,6)$ and $E_{6} \sim G(4,7)$, respectively. Very recently, a natural way of identifying a finite set of letters from $G(4,8)$ has been proposed in Refs. [62-64]. Starting from the eight-particle alphabet thus obtained in the latter paper, here we will establish a connection with five-particle scattering in generic gauge theory. The eightparticle alphabet of Ref. [64] consists of 272 rational and 18 square-root letters and contains both the alphabets of the two-loop maximally-helicity-violating [65] and next-to-

\begin{tabular}{|c|c|c|c|}
\hline Integral & $\begin{array}{c}\text { Number of } \\
\text { variables }\end{array}$ & $\begin{array}{l}\text { Number } \\
\text { of letters }\end{array}$ & $\begin{array}{l}\text { Cluster } \\
\text { algebra }\end{array}$ \\
\hline One-mass hexagon & 4 & 16 & $D_{4}$ \\
\hline Two-mass-easy hexagon & 5 & 24 & $\subset D_{5}$ \\
\hline $\begin{array}{l}\text { Two-mass-hard hexagon, } \\
\text { pentagon with one off-shell leg }\end{array}$ & 5 & 27 & $\lim G(4,8)$ \\
\hline
\end{tabular}
maximally-helicity-violating amplitudes [66] (see also [67-69]). In a first step, we specify the kinematics to describe two adjacent massive legs. We find that this reduces the $G(4,8)$ alphabet to $30+5$ rational and

TABLE III. Six-dimensional integrals and cluster algebras. 
algebraic letters, consistent with the symbol of the sixdimensional two-mass-hard hexagon, as obtained from Ref. [70]. The alphabet we find also applies to nondual-conformal five-particle scattering with one off-shell leg, as the latter can be obtained from the hexagon by a dual conformal transformation. Indeed, we reproduce all letters appearing in the one-loop integrals of Ref. [71], except for $W_{48}$ and $W_{49}$. Taking in addition the limit to massless fiveparticle kinematics, we find 22 letters from the planar pentagon alphabet [72]. These include all one-loop letters except for $W_{31}$ (using the notation of Ref. [73]). Moreover, the reduction of the octagon alphabet also captures some of the additional two-loop letters; upon cyclic symmetrization, we obtain all planar two-loop letters, apart from $W_{31}$. This is very interesting, because $W_{31}$ has been observed to drop out of appropriately defined finite quantities, such as the hard part (after infrared subtraction) of the two-loop $\mathcal{N}=4$ SYM [74,75] and $\mathcal{N}=8$ supergravity [76,77] amplitudes. The same is true for the hard part of the two-loop $q \bar{q} \rightarrow \gamma \gamma \gamma$ amplitude [78,79] and the two-loop five-gluon amplitudes [80-82] in QCD.

Interested experts may find the technical details of the identifications mentioned above in Supplemental Material [83].

Discussion and outlook.-In this Letter, we have provided for the first time evidence that cluster algebras are relevant to Feynman integrals beyond $\mathcal{N}=4 \mathrm{SYM}$.

In particular, we have uncovered that the important class of two-dimensional harmonic polylogarithms corresponds to the $C_{2}$ cluster algebra. This suggests that a number of physical processes, including Higgs plus jet amplitudes in the heavy top quark limit, may be amenable to bootstrap methods [84]. Moreover, we found unexpected adjacency relations that further constrain the function space. By identifying $C_{2}$ as a subspace of the $A_{3}$ cluster algebra, we have shown that these adjacencies are equivalent to the extended Steinmann relations of planar six-particle scattering in $\mathcal{N}=4$ SYM $[9,10]$. Such relations have been used for six- and higher-particle amplitudes (and five-particle scattering with one off-shell leg [71]), but their implications are not yet known for massless five-particle scattering. This makes the adjacency relations we found in a five-particle kinematics all the more interesting. We have demonstrated that the constraints we found significantly reduce the relevant functions space; cf. Table I. This should prove valuable in bootstrap approaches. This is very timely in view of the recent integrability results for form factors in $\mathcal{N}=4$ SYM [41].

We have outlined heuristic procedures for matching Feynman integrals with cluster algebras and found a host of one-loop identifications. This suggests a number of questions. What other processes are related to cluster algebras? What cluster algebras describe the higher-loop versions of the cases discussed here? We leave these questions to future work and hope that our results motivate a systematic taxonomy of the relationship between Feynman integrals and cluster algebras.

In planar $\mathcal{N}=4 \mathrm{SYM}$, there is a direct link between the kinematics and geometry of the scattering process and cluster algebras. This leads us to the most pressing open question: Is there a similar story for generic Feynman integrals?

We thank S. Badger, L. Dixon, P. Mastrolia, A. McLeod, V. Sotnikov, M. Wilhelm and S. Zoia for useful correspondence, N. Henke for discussions, and M. Spradlin for comments on the manuscript. J. M. H. and G. P. thank the Higgs Centre for Theoretical Physics for hospitality during the workshop Cluster Algebras and the Geometry of Scattering Amplitudes in the early stages of this project. This research received funding from the European Research Council (ERC) under the European Union's Horizon 2020 research and innovation program (Grant Agreement No. 725110), Novel structures in scattering amplitudes. G. P. acknowledges support from the Deutsche Forschungsgemeinschaft under Germany's Excellence Strategy EXC 2121 Quantum Universe 390833306.

\section{*chicheri@mpp.mpg.de}

†henn@mpp.mpg.de

*georgios.papathanasiou@desy.de

[1] N. Arkani-Hamed, J. L. Bourjaily, F. Cachazo, S. CaronHuot, and J. Trnka, J. High Energy Phys. 01 (2011) 041.

[2] N. Arkani-Hamed, F. Cachazo, C. Cheung, and J. Kaplan, J. High Energy Phys. 03 (2010) 020.

[3] N. Arkani-Hamed and J. Trnka, J. High Energy Phys. 10 (2014) 030.

[4] N. Arkani-Hamed, J. L. Bourjaily, F. Cachazo, A. B. Goncharov, A. Postnikov, and J. Trnka, Grassmannian Geometry of Scattering Amplitudes (Cambridge University Press, Cambridge, England, 2016).

[5] J. Golden, A. B. Goncharov, M. Spradlin, C. Vergu, and A. Volovich, J. High Energy Phys. 01 (2014) 091.

[6] L. J. Dixon, J. M. Drummond, and J. M. Henn, J. High Energy Phys. 11 (2011) 023.

[7] J. M. Drummond, G. Papathanasiou, and M. Spradlin, J. High Energy Phys. 03 (2015) 072.

[8] S. Caron-Huot, L. J. Dixon, A. McLeod, and M. von Hippel, Phys. Rev. Lett. 117, 241601 (2016).

[9] S. Caron-Huot, L. J. Dixon, M. von Hippel, A. J. McLeod, and G. Papathanasiou, J. High Energy Phys. 07 (2018) 170.

[10] S. Caron-Huot, L. J. Dixon, F. Dulat, M. Von Hippel, A. J. McLeod, and G. Papathanasiou, J. High Energy Phys. 09 (2019) 061.

[11] J. Drummond, J. Foster, and Ö. Gürdoğan, Phys. Rev. Lett. 120, 161601 (2018).

[12] J. Drummond, J. Foster, and Ö. Gürdoğan, J. High Energy Phys. 03 (2019) 086.

[13] L. J. Dixon, J. Drummond, T. Harrington, A. J. McLeod, G. Papathanasiou, and M. Spradlin, J. High Energy Phys. 02 (2017) 137. 
[14] J. Drummond, J. Foster, Ö. Gürdoğan, and G. Papathanasiou, J. High Energy Phys. 03 (2019) 087.

[15] S. Caron-Huot, L. J. Dixon, F. Dulat, M. von Hippel, A. J. McLeod, and G. Papathanasiou, J. High Energy Phys. 08 (2019) 016.

[16] L. J. Dixon and Y.-T. Liu, J. High Energy Phys. 10 (2020) 031.

[17] S. Caron-Huot, L. J. Dixon, J. M. Drummond, F. Dulat, J. Foster, O. Gürdoğan, M. von Hippel, A. J. McLeod, and G. Papathanasiou, Proc. Sci., CORFU2019 (2020) 003 [arXiv:2005.06735].

[18] J. Drummond, J. Henn, G. Korchemsky, and E. Sokatchev, Nucl. Phys. B828, 317 (2010).

[19] N. Berkovits and J. Maldacena, J. High Energy Phys. 09 (2008) 062.

[20] J. M. Drummond, J. M. Henn, and J. Plefka, J. High Energy Phys. 05 (2009) 046.

[21] N. Arkani-Hamed, J. L. Bourjaily, F. Cachazo, and J. Trnka, J. High Energy Phys. 06 (2012) 125.

[22] J. M. Henn, Phys. Rev. Lett. 110, 251601 (2013).

[23] A. B. Goncharov, M. Spradlin, C. Vergu, and A. Volovich, Phys. Rev. Lett. 105, 151605 (2010).

[24] C. Duhr, Annu. Rev. Nucl. Part. Sci. 69, 15 (2019).

[25] J. M. Henn, arXiv:2006.00361.

[26] S. Fomin and A. Zelevinsky, J. Am. Math. Soc. 15, 497 (2002).

[27] S. Fomin and A. Zelevinsky, Inventiones Mathematicae 154, 63 (2003).

[28] A. Berenstein, S. Fomin, and A. Zelevinsky, Duke Math. J. 126, 1 (2005).

[29] S. Fomin and A. Zelevinsky, Compos. Math. 143, 112 (2007).

[30] B. Keller, arXiv:0807.1960.

[31] P. Lampe, Cluster Algebras, 4 December 2013, http://www .math.uni-bielefeld.de/ lampe/teaching/cluster/cluster.pdf.

[32] S. Fomin, L. Williams, and A. Zelevinsky, arXiv:1608.05735.

[33] S. Fomin, L. Williams, and A. Zelevinsky, arXiv:1707.07190.

[34] V. V. Fock and A. B. Goncharov, Ann. Sci. Éc. Norm. Supér. (4) 42, 865 (2009).

[35] D. Parker, A. Scherlis, M. Spradlin, and A. Volovich, J. High Energy Phys. 11 (2015) 136.

[36] K.-T. Chen, Bull. Am. Math. Soc. 83, 831 (1977).

[37] T. Gehrmann, M. Jaquier, E. Glover, and A. Koukoutsakis, J. High Energy Phys. 02 (2012) 056.

[38] C. Duhr, J. High Energy Phys. 08 (2012) 043.

[39] T. Gehrmann, L. Tancredi, and E. Weihs, J. High Energy Phys. 04 (2013) 101.

[40] A. Brandhuber, M. Kostacinska, B. Penante, and G. Travaglini, Phys. Rev. Lett. 119, 161601 (2017).

[41] A. Sever, A. G. Tumanov, and M. Wilhelm, Phys. Rev. Lett. 126, 031602 (2021).

[42] T. Gehrmann and E. Remiddi, Nucl. Phys. B601, 248 (2001).

[43] T. Gehrmann and E. Remiddi, Nucl. Phys. B601, 287 (2001).

[44] S. Di Vita, P. Mastrolia, U. Schubert, and V. Yundin, J. High Energy Phys. 09 (2014) 148.

[45] T. Gehrmann and E. Remiddi, Comput. Phys. Commun. 144, 200 (2002).

[46] At one loop, the subalphabet $\left\{z_{1}, z_{2}, 1-z_{1}, 1-z_{2}, z_{1}+z_{2}\right\}$ is sufficient. It corresponds to the $A_{2}$ cluster algebra.

[47] J. Golden, M. F. Paulos, M. Spradlin, and A. Volovich, J. Phys. A 47, 474005 (2014).
[48] L. J. Dixon, A. J. McLeod, and M. Wilhelm, arXiv:2012.12286.

[49] Q. Jin and G. Yang, Phys. Rev. Lett. 121, 101603 (2018).

[50] More generally, we find that $C_{n}$ can be identified as the parity-invariant surface inside $A_{2 n-1}$ and that the same holds true for $F_{4}$ inside $E_{6}$. Similarly, we have obtained $G_{2}$ and $B_{n}$ from $D_{4}$ and $D_{2 n-1}$, respectively. See also [51].

[51] N. Arkani-Hamed, S. He, and T. Lam, arXiv:2005.11419.

[52] Note, however, that the first entries are $z_{i}$ with $i=1,2,3$, while the adjacency conditions apply to the $1-z_{i}$ entries; i.e., the relations found cannot be interpreted as Steinmann relations in the kinematic space of four-particle scattering with one off-shell leg.

[53] It is very interesting to note that the $A_{3}$ mutations that lie on the parity-invariant surface relate $a_{2 i-1} \leftrightarrow a_{2 i+1}$, i.e., exactly the pairs appearing in the adjacency relations (11) we observe.

[54] J. M. Henn, A. V. Smirnov, and V. A. Smirnov, J. High Energy Phys. 07 (2013) 128.

[55] J. M. Henn, A. V. Smirnov, and V. A. Smirnov, J. High Energy Phys. 03 (2014) 088.

[56] J. Henn, B. Mistlberger, V. A. Smirnov, and P. Wasser, J. High Energy Phys. 04 (2020) 167.

[57] J. M. Henn and V. A. Smirnov, J. High Energy Phys. 11 (2013) 041.

[58] In fact, only eight independent combinations of the letters appear in the one-loop differential equations.

[59] E. Remiddi and J. Vermaseren, Int. J. Mod. Phys. A 15, 725 (2000).

[60] V. Del Duca, C. Duhr, and V. A. Smirnov, J. High Energy Phys. 07 (2011) 064.

[61] V. Del Duca, L. J. Dixon, J. M. Drummond, C. Duhr, J. M. Henn, and V. A. Smirnov, Phys. Rev. D 84, 045017 (2011).

[62] N. Arkani-Hamed, T. Lam, and M. Spradlin, arXiv:1912.08222.

[63] N. Henke and G. Papathanasiou, J. High Energy Phys. 08 (2020) 005.

[64] J. M. Drummond, J. Foster, Ö. Gürdoğan, and C. Kalousios, arXiv:1912.08217.

[65] S. Caron-Huot, J. High Energy Phys. 12 (2011) 066.

[66] C. Zhang, Z. Li, and S. He, Phys. Rev. D 101, 061701 (2020).

[67] S. He, Z. Li, and C. Zhang, arXiv:2009.11471.

[68] J. Mago, A. Schreiber, M. Spradlin, and A. Volovich, J. High Energy Phys. 10 (2020) 128.

[69] S. He and Z. Li, arXiv:2007.01574.

[70] M. Spradlin and A. Volovich, J. High Energy Phys. 11 (2011) 084.

[71] S. Abreu, H. Ita, F. Moriello, B. Page, W. Tschernow, and M. Zeng, J. High Energy Phys. 11 (2020) 117.

[72] T. Gehrmann, J. M. Henn, and N. A. Lo Presti, Phys. Rev. Lett. 116, 062001 (2016);116, 189903(E) (2016).

[73] D. Chicherin, J. Henn, and V. Mitev, J. High Energy Phys. 05 (2018) 164.

[74] S. Abreu, L. J. Dixon, E. Herrmann, B. Page, and M. Zeng, Phys. Rev. Lett. 122, 121603 (2019).

[75] D. Chicherin, T. Gehrmann, J. M. Henn, P. Wasser, Y. Zhang, and S. Zoia, Phys. Rev. Lett. 122, 121602 (2019).

[76] D. Chicherin, T. Gehrmann, J. M. Henn, P. Wasser, Y. Zhang, and S. Zoia, J. High Energy Phys. 03 (2019) 115.

[77] S. Abreu, L. J. Dixon, E. Herrmann, B. Page, and M. Zeng, J. High Energy Phys. 03 (2019) 123. 
[78] S. Abreu, B. Page, E. Pascual, and V. Sotnikov, J. High Energy Phys. 01 (2021) 078.

[79] V. Sotnikov (private communication).

[80] S. Badger, C. Brønnum-Hansen, H. B. Hartanto, and T. Peraro, J. High Energy Phys. 01 (2019) 186.

[81] S. Abreu, J. Dormans, F. Febres Cordero, H. Ita, B. Page, and V. Sotnikov, J. High Energy Phys. 05 (2019) 084.
[82] S. Badger and S. Zoia (private communication).

[83] See Supplemental Material at http://link.aps.org/ supplemental/10.1103/PhysRevLett.126.091603 for technical details of the identifications between one-loop alphabets and cluster algebras.

[84] A. Brandhuber, G. Travaglini, and G. Yang, J. High Energy Phys. 05 (2012) 082. 